\title{
Vortex filamentation: its onset and its role on axisymmetrization and merger
}

\author{
O.U. Velasco Fuentes* \\ Departamento de Oceanografía Física, CICESE, Ensenada, Baja California, México \\ Received 23 February 2004; accepted 4 October 2004 \\ Available online 2 December 2004
}

\begin{abstract}
Vortex filamentation in two-dimensional flows is revisited. Attention is centered on its onset and its role on the axisymmetrization of elongated vortices and on the merger of vortex pairs. The objective is to test two generally accepted hypotheses; namely, that filamentation occurs because a saddle stagnation point enters the vortex and that filaments cause both axisymmetrization and merger. These hypotheses are based on the analysis of the Eulerian flow geometry, i.e., the set of stagnation points and associated streamlines of the instantaneous velocity field. Here, on the contrary, filamentation is described and quantified by analyzing the Lagrangian flow geometry, i.e., the set of hyperbolic trajectories and associated manifolds of the time evolving velocity field. This dynamical-systems approach is applied to the numerical representation of the velocity field obtained by solving the Euler equations with a vortex-in-cell model. Filamentation is found to occur because a stable manifold of a hyperbolic trajectory enters the vortex, and it is unimportant whether the hyperbolic trajectory or the saddle point are inside the vortex or whether they are outside. It is also found that filamentation, although an important part of both axisymmetrization and merger, is not the cause of these processes.
\end{abstract}

(C) 2004 Elsevier B.V. All rights reserved.

Keywords: Vortex filamentation; Axisymmetrization; Merger; Lobe dynamics

\footnotetext{
* Present address: CICESE, P.O. Box 434844, San Diego, CA 92143, USA. Tel.: + 52646 1750500; fax: +526461750547 .

E-mail address: ovelasco@ cicese.mx (O.U. Velasco Fuentes).
} 


\section{Introduction}

Vortex axisymmetrization (the relaxation of elongated vortices towards axial symmetry) and vortex merger (the coalescence of a pair of like-signed vortices into a single structure) are fundamental phenomena in two-dimensional flows and share important features (Melander et al., 1987, 1988). It is well known that in the course of these processes the vortices expel filaments in amounts that depend on the initial conditions (vorticity distribution, aspect ratio of the elongated vortex, intercentroid distance of the vortex pair, etc.). Vortex filamentation is generally considered to occur because a stagnation point of saddle type lies within the vortex (Melander et al., 1987, 1988; Polvani et al., 1989; Mariotti et al., 1994; Cerretelli and Williamson, 2003) and it has been proposed as the cause of axisymmetrization (Melander et al., 1987) and merger (Melander et al., 1988; Cerretelli and Williamson, 2003). These hypotheses are based on comparing the vorticity distribution with the Eulerian flow geometry, which may be defined as the set of stagnation points of saddle type and the associated streamlines as observed in the instantaneous velocity field. One weakness of this approach is that it usually requires the computation of the Eulerian geometry in a moving reference system where the flow appears to be stationary. Such a system, however, does not exist for the flows under study here because they are essentially time dependent. At best, systems can be found where the flow appears to evolve slowly, but the particular system used is always the observer's choice. See Fig. 1, which shows the Eulerian flow geometry for an elliptic vortex and for a pair of vortices in a fixed frame [(a) and (c), respectively] and in a frame rotating with the vortices [(b) and (d), respectively]. Fortunately, the location of a saddle point does not change significantly with any reasonably chosen reference system and this small uncertainty may be considered a minor weakness of the approach. A crucial flaw is, however, that filamentation usually starts well before any saddle point enters the vortex.

Vortex filamentation is a manifestation of fluid advection. Therefore, it will be studied here using methods from the theory of transport in dynamical systems. In particular, we will use the Lagrangian flow geometry, i.e., the set of hyperbolic trajectories and their associated manifolds. Loosely speaking, the hyperbolic trajectory is the path of a fluid particle which attracts one set of particles and repels a different set of particles. Since the hyperbolic trajectory is the path of a material particle, we will refer to this particle as hyperbolic particle. The set of particles, which are attracted towards the hyperbolic particle constitute the stable manifold, and the set of repelled particles constitute the unstable manifold. Here, of course, the terms attract and repel are used only as a description of how the fluid moves in the vicinity of the hyperbolic particle.

The relation between the Lagrangian and Eulerian geometries depends on the way the flow under consideration evolves in time. In steady flows both geometries are identical: the saddle points are hyperbolic trajectories, and the stretching and squeezing streamlines are the unstable and stable manifolds, respectively. In flows that can be considered as a mean steady flow plus a small time-dependent perturbation, the two geometries closely resemble each other: the hyperbolic particle is always close to the saddle point and the manifolds emanating from the hyperbolic trajectories are parallel to the streamlines in the vicinity of the saddle point. Early studies of chaotic advection dealt mostly with flows of this type (e.g., Rom-Kedar et al., 1990). Realistic flows are usually more complicated: they are aperiodic 


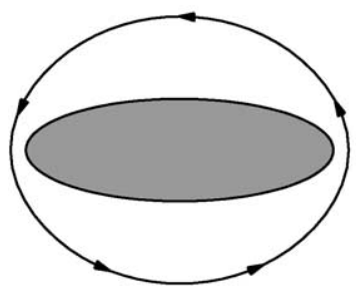

(a)

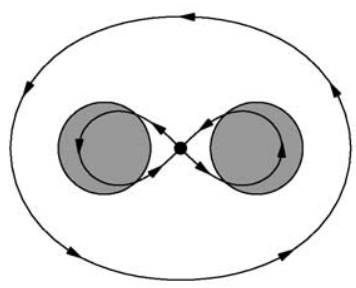

(c)
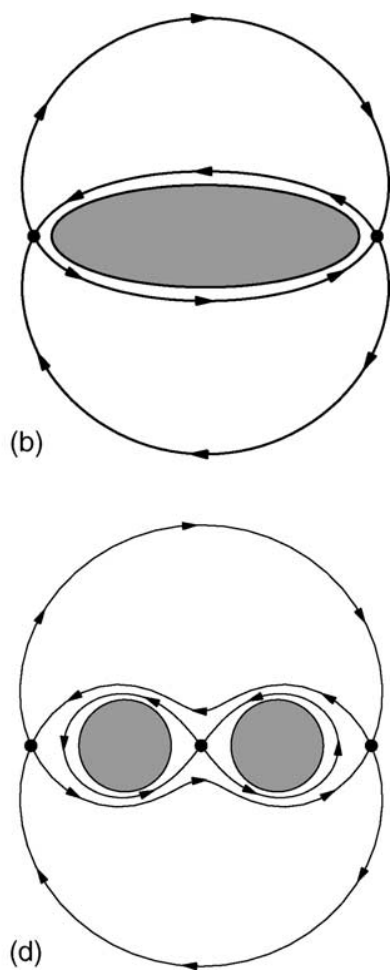

Fig. 1. Stream function of a single elliptic vortex (a and b) and a pair of equal circular vortices (c and d), as observed in a fixed reference frame ( $a$ and c) and in a frame co-rotating with the vortex structure (b and d). The gray areas are the vortices, the black dots indicate the saddle points, and the arrows indicate the direction of particle motion along the streamlines.

and may undergo qualitative changes in the course of the evolution. For instance, vortex merger implies the disappearance of one of the three saddle stagnation points present in the initial condition, and complete axisymmetrization implies the disappearance of the two saddle points. As a consequence the Eulerian flow geometry may differ significantly from the Lagrangian one, and any consequences derived from the former may be misleading.

The existence of stagnation points may facilitate the detection of the Lagrangian flow geometry. Some analytic results guarantee that, in periods of slow evolution, hyperbolic trajectories exist in the neighborhood of the stagnation points (Haller and Poje, 1998; Malhotra and Wiggins, 1998). The Eulerian geometry is thus used as a first step in the construction of the Lagrangian geometry. This approach has been very successful for computing transport templates for vortex-jet and vortex-vortex interaction problems (e.g., Poje and Haller, 1999; Velasco Fuentes, 2001). Therefore, this approach is best suited for achieving the objectives of the present study: (a) to relate the Eulerian and the Lagrangian flow geometries to the onset of filamentation and (b) to determine the role that filaments play in vortex axisymmetrization and vortex merger. 
The rest of the paper is organized as follows. A brief description of the methods is given in Section 2. In Section 3 we study the axisymmetrization of elliptical vortices with nonuniform vorticity profile. Section 4 deals with the merger of vortex pairs, and the conclusions are given in Section 5.

\section{Methods}

\subsection{Numerical solution of the equation of motion}

We will consider the two-dimensional, unforced flow of an ideal fluid. Such system is governed by the Euler equation, which can be written in the following form:

$$
\frac{\partial \omega}{\partial t}+J(\omega, \psi)=0
$$

where $\omega=\partial v / \partial x-\partial u / \partial y$ is the vorticity, and $\psi$ is the stream function. Eq. (1) is solved using the vortex-in-cell method (VIC) as implemented in Velasco Fuentes (2001) (see also Hockney and Eastwood (1981), for a general account of particle methods). The numerical domain is a square, uniform grid of $256 \times 256$ points with free-slip boundary conditions. In the simulations of vortex axisymmetrization there are 12 grid points per mean vortex radius, and the elliptic patch of vorticity is represented by about 80,000 point vortices. In the simulations of vortex merger there are ten grid points per vortex radius, and each circular patch of vorticity is represented by about 35,000 point vortices. Some calculations regarding the accuracy of the simulations may be found in the Appendix A.

\subsection{Computation of particle trajectories}

Particle trajectories are the solutions of the following pair of ordinary differential equations:

$$
\frac{\mathrm{d} x}{\mathrm{~d} t}=\frac{\partial \psi(x, y, t)}{\partial y}, \quad \frac{\mathrm{d} y}{\mathrm{~d} t}=-\frac{\partial \psi(x, y, t)}{\partial x},
$$

where $\psi$ is the stream function obtained by numerically solving Eq. (1) with the VIC method. Hence, Eq. (2) must be integrated when the right-hand side is known at discrete spatial and temporal grid points only. The database consists of $N+1$ slices of data, where the $n$th slice is the solution computed at time $t_{n}=n \Delta t$, for $n=0,1, \ldots, N$. Each slice is a $2 \mathrm{D}$ array of data defining the stream function on $M \times M$ grid points. In this study, $N=1000, \Delta t \approx \tau / 125$ (where $\tau$ is the eddy turnover time), and $M=256$.

The integration of Eq. (2) thus requires interpolation in three dimensions to find the velocity at arbitrary points $(x, y, t)$. For consistency, the interpolation in space is made with the same biquadratic scheme used in the VIC model. The time integration is made with a second-order Runge-Kutta scheme, therefore, even though $\psi$ is output at every time step available in the VIC model, the data slices are interpolated by the timeintegrator. 


\subsection{Finding hyperbolic trajectories}

Since the flow is essentially time dependent, the relation between the trajectories of fluid particles (Lagrangian dynamics) and the geometry of the instantaneous velocity field (Eulerian flow) is not straightforward. We expect, however, that if the saddle point exists long enough and the velocity field around it changes slowly then there is a hyperbolic particle in its neighborhood. This can be proved rigorously; see Haller and Poje (1998) and Malhotra and Wiggins (1998) for the theory, and Velasco Fuentes (2001) for an example of the use of the criteria. Usually this condition is satisfied uninterruptedly during vortex axisymmetrization, and it is satisfied at various stages during vortex merger. When the condition is satisfied, the position of the hyperbolic particle and its manifolds at any given time $t$ can be computed by the method described below. With suitable variations, this method has been extensively used for velocity fields defined analytically or given as data sets, and with periodic, quasi-periodic or arbitrary time dependence (e.g., Rom-Kedar et al., 1990; Beigie et al., 1994; Malhotra and Wiggins, 1998; Velasco Fuentes, 2001).

The starting point of the analysis is the stream function $\psi$ computed by the VIC model (e.g., Fig. 1a and c). The stream function $\Psi$ observed in a system that rotates with the vorticity distribution is given by the simple transformation $\Psi=\psi+\frac{1}{2} \Omega\left[\left(x-x_{\mathrm{c}}\right)^{2}+\left(y-y_{\mathrm{c}}\right)^{2}\right]$, where $\Omega$ is the angular velocity and $\left(x_{\mathrm{c}}, y_{\mathrm{c}}\right)$ is the observed center of rotation. Because of the initial symmetry, the center of rotation remains at the vortex center when there is one vortex and at the middle point between the vortices when there are two of them. A good estimation of $\Omega$ is obtained making any of the following assumptions: (a) a single, elongated vortex is taken to be an elliptic patch of uniform vorticity; or (b) a pair of vortices is taken to be a pair of point vortices. In either case the angular velocity $\Omega$ is evaluated from the corresponding analytic expression: (a) $\Omega=a b \omega /(a+b)^{2}$, where $\omega$ is the relative vorticity and $a$ and $b$ the semi-axes of the ellipse; or (b) $\Omega=\Gamma /\left(\pi d^{2}\right)$, where $\Gamma$ is the circulation of each vortex and $d$ is the intercentroid distance.

The second step is to determine the geometry of the co-rotating stream function $\Psi$; that is to say, locate the saddle points and the streamlines associated to them. A wide variety of methods and tools for obtaining these geometric elements from a numerically generated flow field are available (see Velasco Fuentes, 2001 for the implementation used here).

Finally, the stable manifold is obtained computing the evolution, from time $t+\Delta t$ to time $t$, of a short line, which crosses the saddle point of $\Psi(x, y, t+\Delta t)$ in the squeezing direction; and the unstable manifold is obtained computing the evolution, from time $t-\Delta t$ to time $t$, of a short line which crosses the saddle point of $\Psi(x, y, t-\Delta t)$ in the stretching direction (see Fig. 2). The position of the hyperbolic particle is given by the intersection of the manifolds.

\section{Evolution of elongated vortices}

In the last three decades much work has been done on the evolution of single, unsteady vortices. Various settings have been considered: a perturbed Kirchhoff's vortex (Polvani et al., 1989), nonuniform elliptical vortices (Melander et al., 1987; Koumoutsakos, 1997), and vortices with various vorticity distributions embedded in a background flow (Mariotti et 


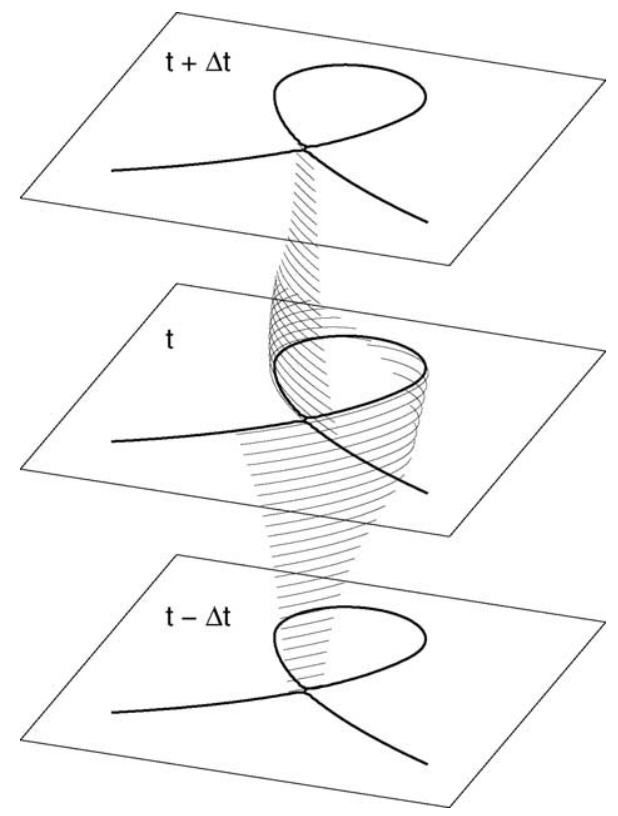

Fig. 2. Numerical computation of the manifolds at time $t$. Small line segments are placed along the stretching and squeezing directions of the saddle point at times $t-\Delta t$ and $t+\Delta t$, respectively. The evolution of this segments toward time $t$ give the unstable and stable manifolds. The intersection of the segments give the position of the hyperbolic particle.

al., 1994). Filamentation of the vortex has usually been observed, sometimes accompanied by a tendency towards axial symmetry. It has been proposed that filamentation occurs because there is a saddle point on the boundary or inside the vortex, and that the filaments drive the evolution towards axial symmetry (Melander et al., 1987). In this section, we will test this hypothesis for Kirchhoff vortices and for elliptic vortices with nonuniform vorticity distributions.

\subsection{Filamentation of a perturbed Kirchhoff's vortex}

Fig. 3 shows the evolution of a Kirchhoff vortex in the unstable regime (initial aspect ratio is 3.33). A perturbation has been prescribed as follows: the vortex contour has a deformation of wave number 3 and an amplitude of $2 \%$ relative to the length of the mean radius. Polvani et al. (1989) used the same conditions in their study of the onset of filamentation. The instability produces the ejection of a thin vortex filament and a slight change of shape but there is no tendency towards axial symmetry. In fact, the vortex is less axisymmetric in the last stage shown than in its initial condition (see column a).

During the whole evolution the Eulerian and the Lagrangian flow geometries (columns $b$ and c, respectively) show a remarkable resemblance. Consequently, the hyperbolic particle and the saddle point are closely located, as it is shown in Fig. 4. Such a high similarity 


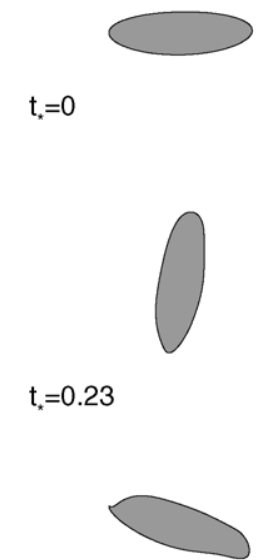

$\mathrm{t}_{\star}=0.45$

(a)

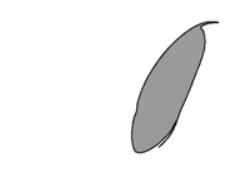

$t_{\star}=0.68$
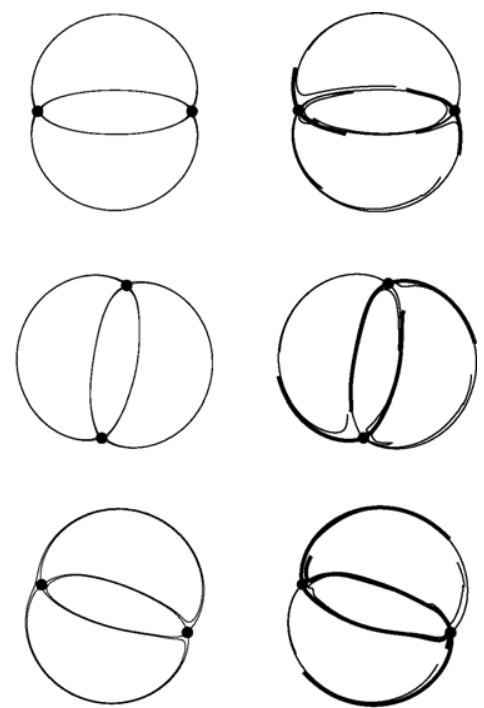

(b)
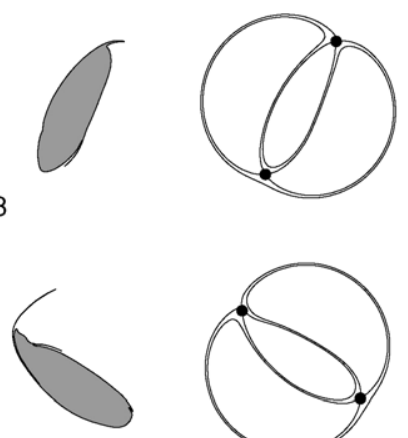

$t_{\star}=0.9$
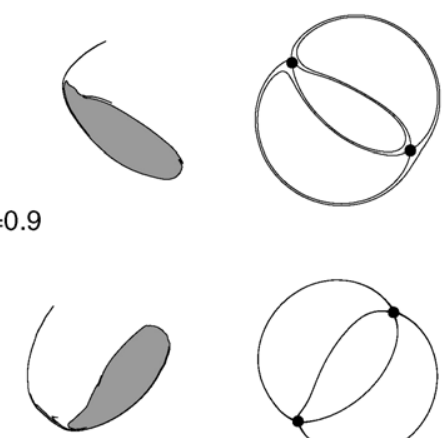

$t_{*}=1.13$

(a)

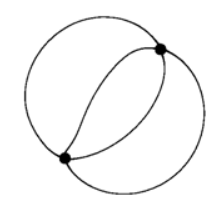

(b)
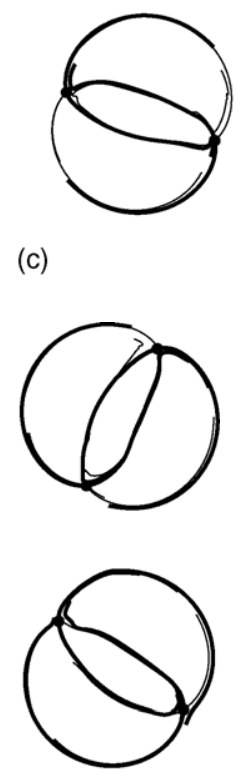

(c)

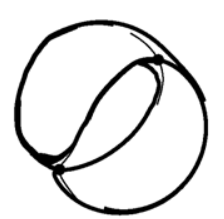

(c)

Fig. 3. Time evolution of a perturbed Kirchhoff vortex (aspect ratio: 3.3; perturbation wave number: 3; perturbation amplitude: $2 \%$ ). (a) Vortex contour, (b) co-rotating stream function, and (c) stable and unstable manifolds (thin and thick lines, respectively). The time $t_{*}$ is given in units of the rotation period of the unperturbed vortex. 


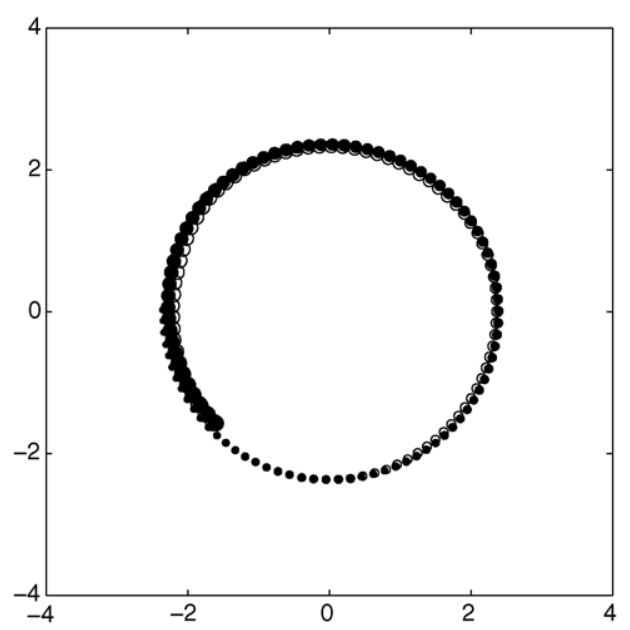

Fig. 4. Path of the saddle point (open circles) and trajectory of the hyperbolic particle (filled circles) during the evolution of the Kirchhoff vortex shown in Fig. 3. The size of the circles increases with the time elapsed.

between the two geometries is a consequence of the flow being always close to a steady solution of the equations of motion.

Subtle differences exist, however, and these are important in relation to the hypothesis that we want to test. Fig. 5 shows a detail of the vortex and the corresponding Eulerian and Lagrangian flow geometries when the saddle point enters the vortex. At this moment the filamentation of the vortex is well under way; i.e., it started when the saddle point was still out of the vortex. Notice also that the vortex contour runs fairly parallel to the unstable manifold, showing that filamentation is governed by the Lagrangian geometry.

\subsection{Axisymmetrization of nonuniform vortices}

A systematic analysis of the filamentation was performed with vortices of vorticity distributions taken from the following family

$$
\omega= \begin{cases}\omega_{0}\left[1-\left(\frac{r}{a}\right)^{n}\right] & \text { for } r<a, \\ 0 & \text { for } r>a,\end{cases}
$$

where $\omega_{0}$ is the peak value of the vorticity, $r$ the distance to the vortex centre and $n$ is an integer. The vorticity profiles considered here are (a) $n=6$ (steep) and (b) $n=2$ (smooth). The initial aspect ratio $\left(e_{0}\right)$ of the vortices was varied in the range $1-3$, which corresponds to the stable regime of Kirchhoff vortices. Note, however, that the vortices given by (3) have nonuniform vorticity (except for $n=\infty$ ), and they are unsteady for all $e_{0} \neq 1$.

In each numerical experiment we determined the locations of hyperbolic particles $H$ (which are intrinsic properties of the flow, and therefore frame independent) and of saddle stagnation points $S$ (which depend on the choice of co-moving frame). In addition, we computed two diagnostic parameters for the approximately elliptic patch of vorticity that survives the filamentation process: area $\left(A_{*}\right)$ and aspect ratio $\left(e_{*}\right)$. Fig. 6 shows results for vortices 


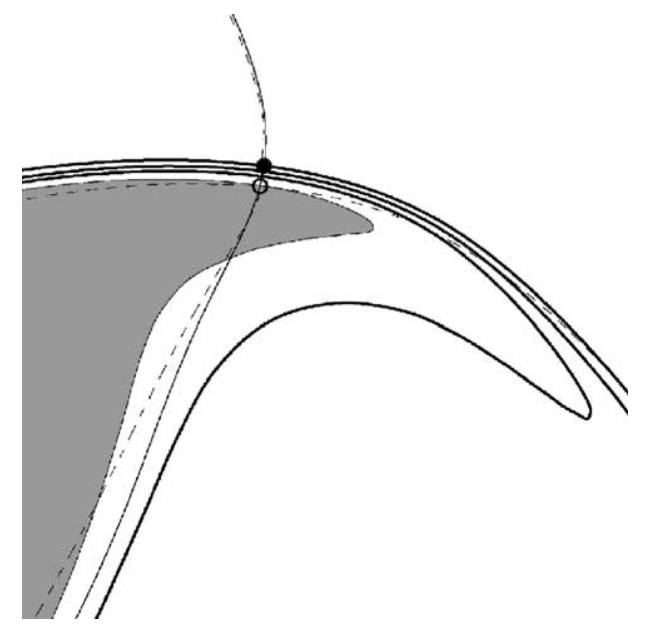

Fig. 5. Detail of the Eulerian and Lagrangian flow geometries at time $t_{*}=0.62$ for the Kirchhoff vortex shown in Fig. 3. This figure shows the hyperbolic particle (black dot) with its manifolds (stable: thin, continuous line; unstable: thick, continuous line); and the saddle point (open circle) with its associated streamlines (broken lines). The gray area is the vortex.

with steep vorticity profile $(n=6)$; results for vortices with smooth vorticity profile $(n=2)$ are qualitatively similar. $A_{*}$ and $e_{*}$ are the quasi-steady values measured at a late stage (i.e., when the vortex ceased to undergo noticeable changes) and are given as a fraction of the corresponding value in the initial condition. Take for instance the vortex with $e_{0}=3$. The values of $A_{*}$ and $e_{*}$ are about 0.8 and 0.65 , respectively, which means that at the final stage the vortex preserves only $80 \%$ of its original area and its aspect ratio decreases to $0.65 \times 3 \approx 2$. Fig. 6 also shows values of $H$ and $S$ in the initial condition. The values are scaled by the length of the semi-major axis of the vortex, so that values larger than one indicate that the point is outside the vortex and values lower than one indicate that the point is inside the vortex.

Analyzing the value of $S$ in combination with the value of $A_{*}$ for a particular initial condition gives an indication of the influence of the initial location of the saddle point on the latter occurrence of filamentation. Fig. 6 shows that vortices with $1.5<e_{0}<2$ have $S>1$ (i.e., the saddle point lies outside the vortex) and yet $A_{*}<1$ (i.e., the area decreases through filamentation). The same happens with the hyperbolic particle: in the interval $1.5<e_{0}<2.75$ the hyperbolic particle lies outside the vortex and yet the area decreases through filamentation.

In a similar way, analyzing the value of $A_{*}$ in combination with the value of $e_{*}$ for a particular initial condition gives an indication of the relation between filamentation (a decrease in $A_{*}$ ) with axisymmetrization (a decrease in $e_{*}$ ). Fig. 6 shows that vortices with $1<e_{0}<1.5$ have $A_{*}=1$ (i.e., there is no filamentation) and yet $e_{*}<1$ (i.e., the aspect ratio decreased during the evolution).

We may, therefore, conclude the following: (a) the saddle point being inside the vortex is a sufficient condition for filamentation, but it is not a necessary one and (b) filamentation is a sufficient condition for decrease of aspect ratio, but it is not a necessary one. 


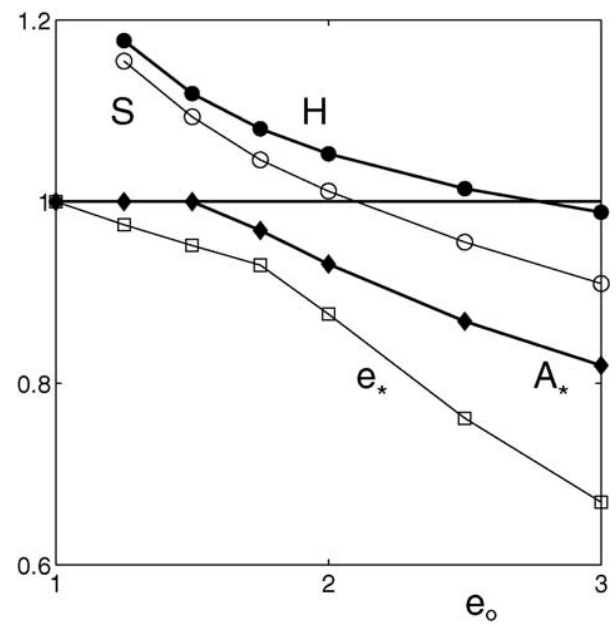

Fig. 6. Diagnostic parameters for an elliptic vortex of nonuniform vorticity as a function of the initial aspect ratio $e_{0}$. The figure shows final values of the vortex area $A_{*}$ (filled diamonds) and the aspect ratio $e_{*}$ (open squares); and the initial relative position of the hyperbolic particle $H$ (filled circles) and the saddle point $S$ (open circles). Values of $A_{*}$ and $e_{*}$ are relative to their initial values; values of $H$ and $S$ are relative to the value of the vortex semi-major axis at $t_{*}=0$ (see text).

\section{Merger of pairs of equal vortices}

The interaction of two like-signed vortices has been the subject of intense research for the last three decades. Most studies have dealt with the highly idealized situation of two circular vortices of equal size and strength. Three flow regimes have been identified in this setting, depending on the initial vortex separation $d_{*}=d / R$ (where $d$ is the intercentroid distance and $R$ is the vortex radius). These regimes are the following: (a) vortices located far apart circle around each other endlessly without undergoing large deformations, (b) vortices located at an intermediate distance circle around each other while exchanging some vorticity, and (c) vortices located at a distance smaller than a certain critical value merge into a single vortex (e.g., Melander et al., 1988). The existence of these regimes has been explained invoking the Eulerian flow geometry (Melander et al., 1988; Cerretelli and Williamson, 2003). According to this hypothesis the evolution depends on how the initial vorticity field is located with respect to the separatrices of the co-rotating stream function (e.g., Fig. 1d): (a) if the vortices are confined inside the inner, eight-shaped separatrix they rotate around each other without undergoing large deformations or merger; (b) if the vortices overstep the inner separatrix but do not go beyond the middle, peanutshaped separatrix they rotate around each other, exchange some vorticity, but do not merge; and finally (c) if the vortices extend beyond the middle separatrix they expel filaments and merge. Melander et al. (1988) proposed this hypothesis simply as an heuristic tool to explain the vortex interaction. Although it has been shown to be inaccurate for various vorticity distributions (Velasco Fuentes, 2001), the same hypothesis has been recently recovered and it has been proposed as the physical mechanism for merger (Cerretelli and 
Williamson, 2003). Hence we will analyze below the relation of the Eulerian and Lagrangian geometries with the different regimes and, especially, the role of filamentation on merger.

Fig. 7 shows the evolution of a pair of equal vortices in the merger regime $\left(d_{*}=3.0\right)$. Column (a) shows the vortex patches, column (b) shows the Eulerian flow geometry as observed in a co-rotating system and column (c) shows the Lagrangian flow geometry. A comparison of frames (a) and (b) in the initial condition $\left(t_{*}=0\right)$ reveals very clearly that the vortices are contained within the middle, peanut-shaped separatrix; therefore, if the hypothesis stated above were correct, they should not merge. The evolution, however, is quite different: the vortices start to merge almost immediately.

Notice that there are large differences between the Eulerian and Lagrangian flow geometries during most of the evolution. These differences arise because at some stage the flow is far from any steady solution of the equations of motion. This far-from-steady stage is the merger event itself, which occurs in a time scale of the order of the eddy turnover time.

The hyperbolic particle and the saddle point are located close to each other during the early stages of the evolution (see Fig. 8). But they separate as soon as merger starts (around
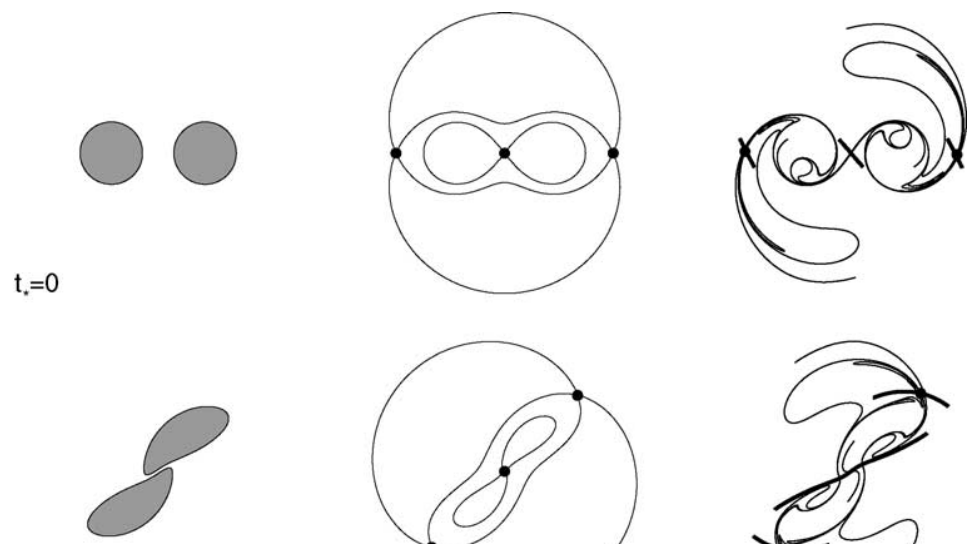

$t_{*}=0.64$
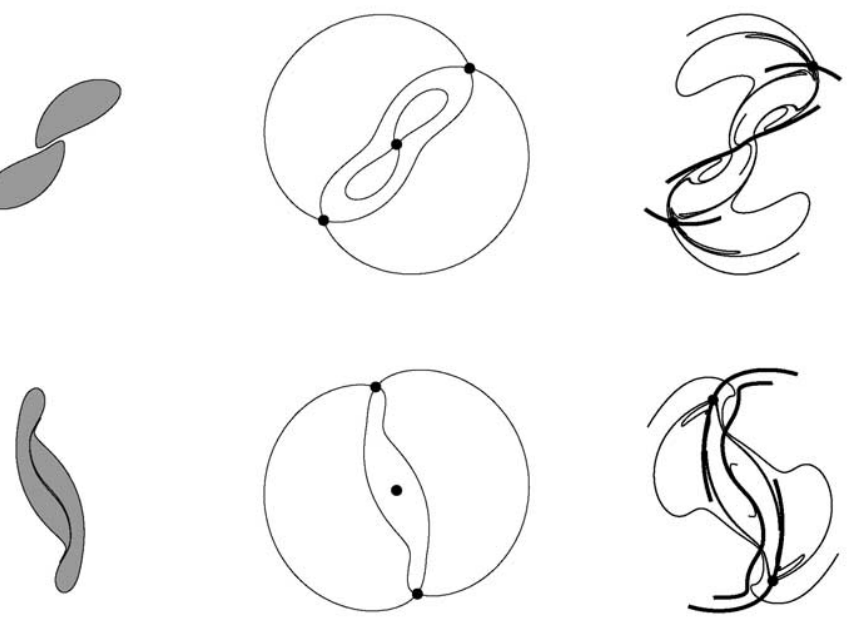

(a)

$$
t_{t}=1.27
$$

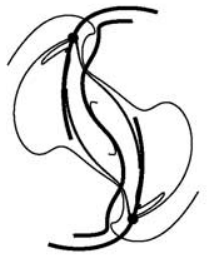

(c)

Fig. 7. Same as Fig. 3 but now for a pair of Rankine vortices (initial separation $d_{*}=3$ ). The time $t_{*}$ is given here in units of the eddy turnover time. 

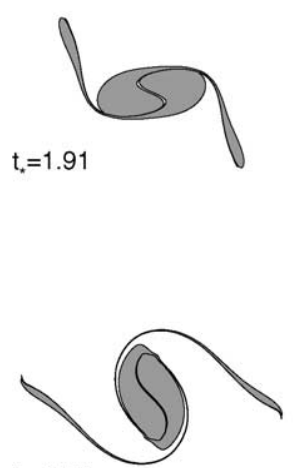

$t_{*}=2.55$

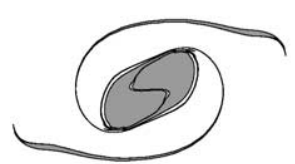

$\mathrm{t}_{\star}=3.18$

(a)
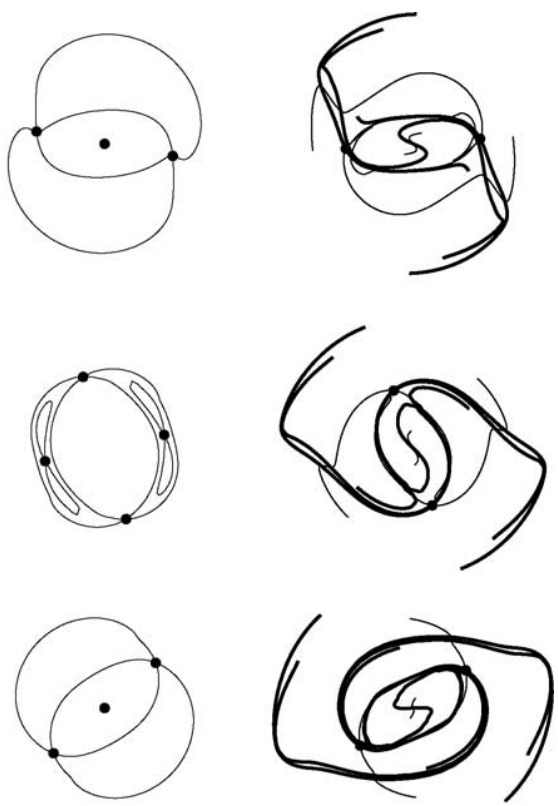

(b)

(c)

Fig. 7. (Continued).

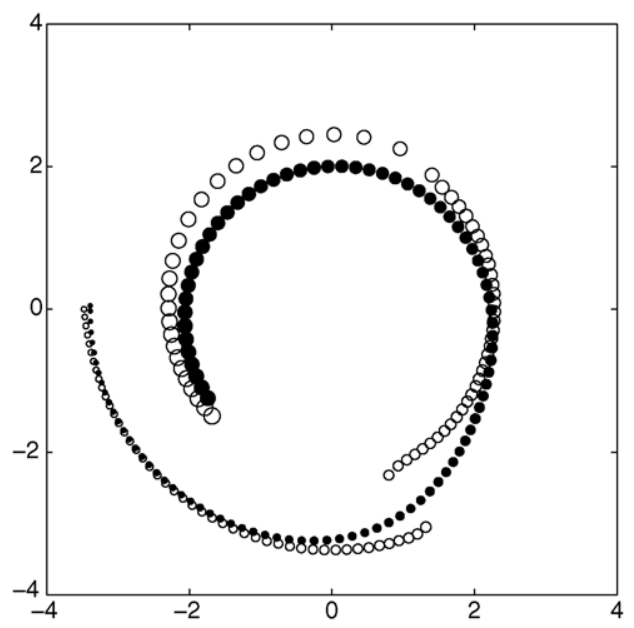

Fig. 8. Same as Fig. 4 but now for the pair of Rankine vortices shown in Fig 7. 
time $t_{*}=0.7$, given in units of the eddy turnover time) and do not approach each other again until the late stages of the evolution (around time $t_{*}=3$ ), when the newly formed vortex stabilizes as an elongated vortex.

\subsection{Lagrangian geometry and regime transitions}

The results of the previous section show that the relative location of the vortices with respect to the Eulerian flow geometry does not predict the outcome of the interaction. In this section, we analyze the Lagrangian flow geometry as the vortices change from one regime to another in order to see if this geometry provides a clue about the outcome of the interaction. The following conventions and definitions will facilitate the exposition below. The hyperbolic particles are denoted by a letter $p$, and a subscript distinguishes each of the three ( 0 for the one in the middle, 1 for the one on the left and 2 for the one on the right). The manifolds are denoted by a letter $W$, the subscript indicates to which hyperbolic particle it belongs to, and the superscript indicates if it is stable $(s)$ or unstable $(u)$. The intersection $P$ of two manifolds is said to be a primary intersection if the manifolds, say $W_{1}^{\mathrm{u}}$ from $p_{1}$ to $P$ and $W_{2}^{\text {s }}$ from $p_{2}$ to $P$, intersect only at $P$.

Fig. 9 shows the Lagrangian flow geometries for vortices in each regime: (a) elasticinteraction, $d_{*}=3.45$; (b) exchange, $d_{*}=3.35$; and (c) merger, $d_{*}=3.25$. Only short segments of the manifolds are shown so that the flow geometry can be clearly observed. Of particular interest are the primary intersections of $W_{1}^{\mathrm{s}}$, with either $W_{1}^{\mathrm{u}}$ or $W_{2}^{\mathrm{u}}$, that are located on the line $p_{1} p_{2}$ or on the perpendicular to that line at $p_{0}$. We will refer to these as symmetric primary intersections (SPI). In the elastic-interaction regime (Fig. 9a) the SPI of $W_{1}^{\mathrm{s}}$ with $W_{2}^{\mathrm{u}}$ is closer to $p_{1}$ than the SPI of $W_{1}^{\mathrm{s}}$ with $W_{1}^{\mathrm{u}}$ (not shown in the figure). This was to be expected because as $d_{*} \rightarrow \infty$ the flow becomes steady and $W_{1}^{\mathrm{s}}$ coincides with $W_{2}^{\mathrm{u}}$ to form the middle separatrix. The situation changes as the flow enters the exchange regime (Fig. 9b), for in this case the SPI of $W_{1}^{\mathrm{s}}$ with $W_{1}^{\mathrm{u}}$ is closer to $p_{1}$ than the SPI of $W_{1}^{\mathrm{s}}$ with $W_{2}^{\mathrm{u}}$. There is thus a qualitative change in the Lagrangian geometry in the transition from the elastic-interaction regime to the exchange regime. Finally, in the merger regime, Fig. 9c, we observe the same qualitative behaviour of the manifolds as in the elastic-interaction regime (N.B. the apparent crossing of $W_{1}^{\mathrm{u}}$ and $W_{2}^{\mathrm{u}}$ is the result of the finite width of the lines: two unstable manifolds can not intersect). Since there is no qualitative difference between the two geometries, it can be concluded that the exchange regime and the merger regime develop initially in a qualitatively similar way. Note also that $W_{1}^{\text {s }}$ does not enter the vortices; therefore no filamentation is involved in the early stages of the evolution, which are characterized by a mutual approach of the vortices. This result is confirmed by the time evolution of the minimal distance between the vortices and of the length of the vortex contours (Fig. 10). The distance between the vortices decreases as soon as the simulation starts, and is reduced to less than $10 \%$ of its initial value in a time $t_{*}=0.5$ for vortices with initial separation $d_{*}=3.0$, and in a time $t_{*}=0.8$ for vortices with $d_{*}=3.25$. In contrast, the length of the vortex contour grows slowly at first and rapidly when filamentation starts (around $t_{*}=0.5$ for $d_{*}=3.0$, and around $t_{*}=0.8$ for $d_{*}=3.25$ ). The start of vortex merger thus precedes the start of filamentation (cf. Cerretelli and Williamson (2003), who attributed the vortex approach to filamentation). 

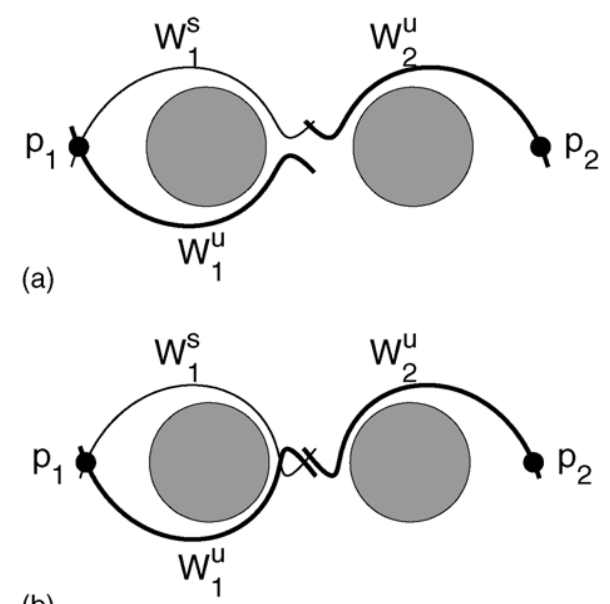

(b)

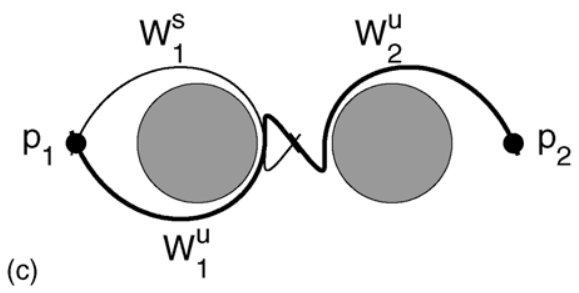

Fig. 9. The Lagrangian flow geometry at time $t_{*}=0$ for a pair of Rankine vortices in (a) the elastic-interaction regime $\left(d_{*}=3.45\right)$, (b) the exchange regime $\left(d_{*}=3.35\right)$, and (c) the merger regime $\left(d_{*}=3.25\right)$. The vortices are represented by gray circles; the stable $\left(W^{\mathrm{s}}\right)$ and unstable $\left(W^{\mathrm{u}}\right)$ manifolds are represented by thin and thick lines, respectively; and the hyperbolic particles $\left(p_{1}\right.$ and $\left.p_{2}\right)$ are indicated by black dots.

\subsection{Quantification of vortex filamentation}

The Lagrangian flow geometry is essentially time dependent, but the properties of the stable and unstable manifolds enable us to draw conclusions on the basis of the flow geometry observed at a given time. In particular, note that the stable and unstable manifolds of hyperbolic particles are invariant surfaces and particles cannot cross them (e.g., Malhotra and Wiggins, 1998). Therefore, a patch of fluid located on one side with respect to a manifold will remain on that side during the whole evolution. The use of this property in the analysis of the intersections of the stable manifolds with the vortex enables us to identify and quantify the areas which are expelled from the vortex. Fig. 11a shows the manifolds of the middle hyperbolic particle $p_{0}$. Particles which are to the right of the manifold (when looking in the flow direction indicated by the arrows) are transported towards the other vortex. Fig. 11b shows the manifolds of the hyperbolic particle $p_{1}$. Particles which are to the right of the manifold are expelled from the vortex to form the spiral arms.

The amount of area exchanged between the vortices $\left(A_{*}^{\mathrm{e}}\right)$ or detrained in filaments $\left(A_{*}^{\mathrm{d}}\right)$ has been computed for initial conditions in the range $2<d_{*}<3.5$ (Fig. 12). This mass, denoted by $A_{*}$, is given as a fraction of the initial mass of the vortex. When the vortices are in 


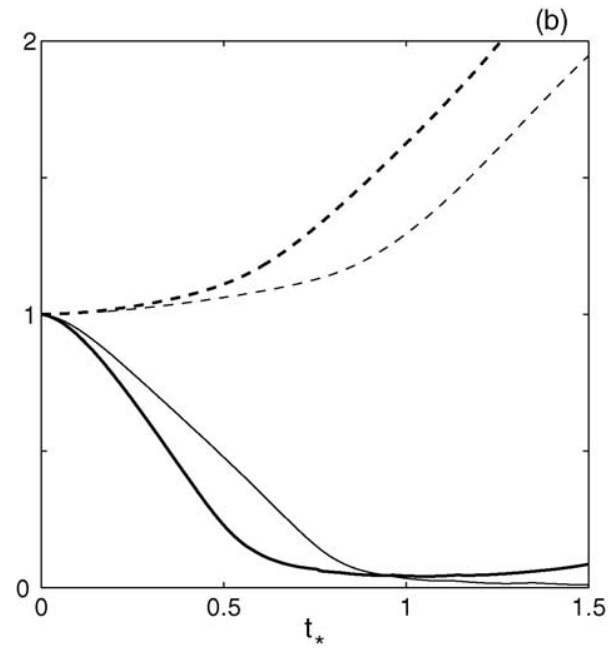

Fig. 10. Evolution of the minimal distance between the vortices (continuos line) and the length of the vortex contour (broken line) in two cases of vortex merger $\left(d_{*}=3.0\right.$ : thick lines; and $d_{*}=3.25$ : thin lines). Lengths are given relative to their initial values, and time is given in units of the eddy turnover time.

touch $\left(d_{*}=2\right)$ the detrained area $A_{*}^{\mathrm{d}}$ is about $8 \%$; and as $d_{*}$ increases, $A_{*}^{\mathrm{d}}$ also increases until it reaches a maximum at $d_{*} \approx 2.9$, when the vortices loose $20 \%$ of their mass. These values agree well with the results of Waugh (1992), who computed the merger efficiency (i.e., the area that is not detrained) to be about $90 \%$ at $d_{*}=2$ and $78 \%$ at $d_{*}=2.9$. As $d_{*}$ further increases $A_{*}^{\mathrm{d}}$ decreases until it becomes zero at $d_{*} \approx 3.35$. Note that for values slightly

(a)
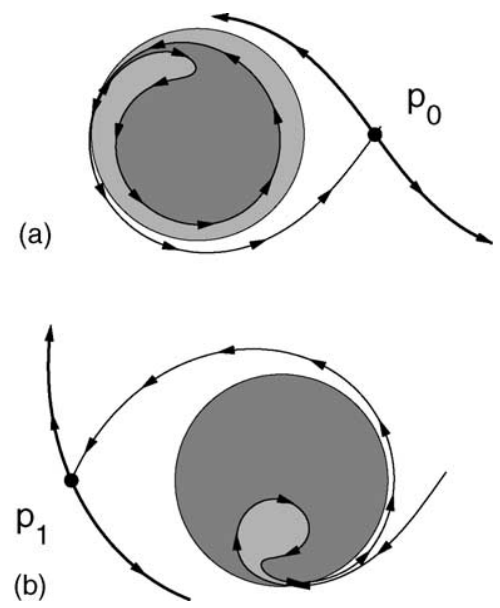

Fig. 11. Evaluation of mass transport. The darker gray areas remain with the vortex, the lighter areas are either (a) exchanged with the vortex partner or (b) expelled to the ambient fluid. The stable and unstable manifolds are represented by thin and thick lines, respectively, and the hyperbolic points are indicated by black dots. 


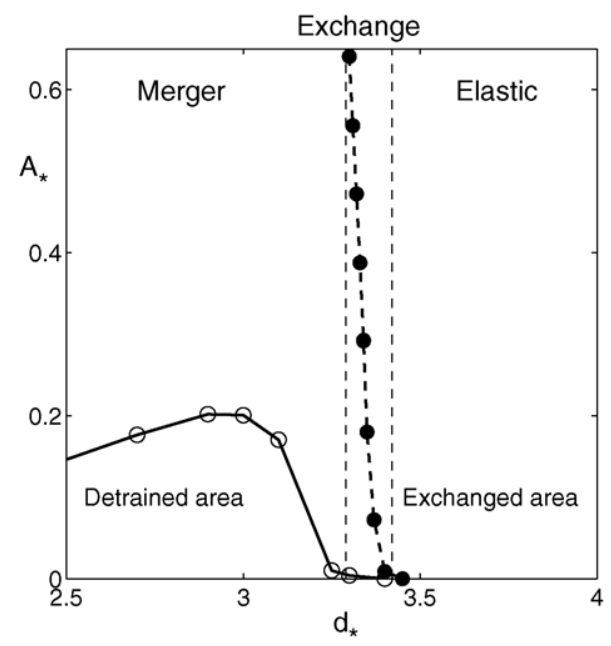

Fig. 12. Area expelled (continuous line) and exchanged (broken line) by Rankine vortices as a function of the initial intercentroid distance $\left(d_{*}\right)$. Values are given as a fraction of the total area in the initial vortex.

above the critical distance for merger the amount of mass expelled is small but different from zero. The area exchanged by the vortices $\left(A_{*}^{\mathrm{e}}\right)$ increases from zero at $d_{*}=3.4$, which is by definition the boundary between the exchange and the elastic-interaction regimes, to more than $60 \%$ at $d_{*}=3.3$, the critical distance for merger.

The same calculations were done with the vortices used by Melander et al. (1988):

$$
\omega=\omega_{0} \begin{cases}1-\exp \left(-\kappa \frac{2 R}{r} \exp \left(\frac{1}{r / 2 R-1}\right)\right) & \text { for } r<2 R, \\ 0 & \text { for } r>2 R,\end{cases}
$$

where $\kappa=\exp (2) \log (2) / 2$ and $R$ is the radius where the vorticity reaches $50 \%$ of its peak value. This equation gives a vorticity profile with an almost conical shape (except that it is smoothly connected to the extreme values). We have chosen it out of the family given by Melander et al. $(1987,1988)$ because it was the one they used when applying the Eulerian geometry to explain vortex axisymmetrization and merger. Below we will refer to them as MMZ vortices.

Defining the vortex radius for an arbitrary vorticity profile is not trivial (e.g., Mitchell and Driscoll, 1996). Natural choices for the profile given by (4) are the radius $r_{\max }$ where the highest flow speed is achieved or the radius $r_{0}$ where the vorticity becomes zero. Note that in Eq. (4) $r_{\max } / r_{0}=0.57$, which is substantially smaller than the corresponding values for Eq. (3), where $r_{\max } / r_{0}=1,0.91,0.82$, for $n=\infty, 6,2$, respectively. Since we have used a pragmatic definition of vortex filament (a region of a vortex which is expelled from it and subsequently undergoes stretching in one direction and squeezing in another), the definition of the vortex radius naturally affects the definition and quantification of the filaments.

If we take $r_{\max }$ as the vortex radius and define "filamentation" as the expulsion of filaments of fluid coming from the region $r<r_{\max }$, then there are initial conditions in which MMZ vortices merge without expelling any filaments. The maximum amount of mass 


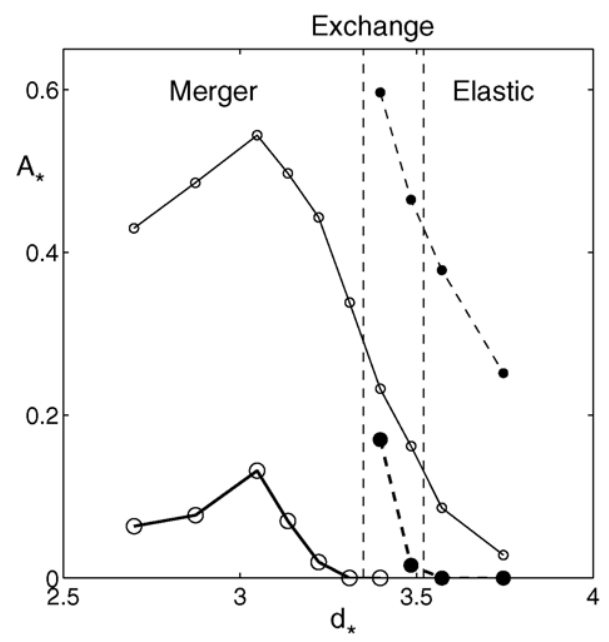

Fig. 13. Same as Fig. 12, but now for the smooth-profile vortices used by Melander et al. (1988). Thick lines and large markers show values obtained when $r_{\max }$ is used as the vortex radius, thin lines and small markers correspond to using $r_{1}$ (see text).

expelled by these vortices (about 15\%) is smaller, but comparable, to the mass expelled by Rankine vortices. In contrast, the amount of mass transported in the exchange regime is considerably different: less than $20 \%$ in MMZ vortices and over $60 \%$ for Rankine vortices.

Instead of using the radius of zero vorticity $r_{0}$ as a second definition of vortex radius, we will use the radius $r_{1}$ where the vorticity reaches $1 \%$ of its peak value (note that $r_{1} / r_{0}=$ 0.83). If we define "filamentation" as the expulsion of filaments of fluid coming from the region $r<r_{1}$, then there are initial conditions in which vortices expel considerable amounts of filaments but do not merge (see Fig. 13). With the new definition the maximum values of the mass expelled during merger and of that transported during exchange rise considerably (to about 50 and $60 \%$, respectively). Note also that the boundary between the exchange and the elastic-interaction regimes change with the definition of the vortex radius.

\section{Conclusions}

Vortex filamentation has been reviewed using the theory of transport in dynamical systems. From this viewpoint filamentation is a manifestation of lobe shedding; therefore, it occurs because the stable manifold of a hyperbolic particle enters the vortex. The hyperbolic trajectory itself may, or may not, enter the vortex; and the same is true for any saddle point (bearing in mind that their locations depend on the reference frame).

The tendency towards axial symmetry of elliptic vortices increases with increasing smoothness of the vorticity profile. For example, a Kirchhoff vortex with aspect ratio $e=3$ preserves that aspect ratio during its evolution; vortices with a steep vorticity profile $[n=6$ in (3) $]$ change from $e=3$ to $e=2$, and vortices with smooth vorticity profile $[n=2$ in (3)] settle at around $e=1.3$. This decrease of aspect ratio is usually accompanied by 
filamentation, but we have also observed that a small decrease of aspect ratio may occur without filamentation taking place.

The regimes of the vortex-pair interaction do not depend on the position of the vortices with respect to the separatrices of the Eulerian flow as proposed in previous works (see Melander et al., 1988; Cerretelli and Williamson, 2003). Similarly, the regimes transitions are not related in an obvious way to changes in the topology of the Lagrangian flow geometry.

In all simulations, merger was accompanied by filamentation, but in some of them filamentation did not lead to merger. It was also observed that, at least for vortices with step-like vorticity profile, merger starts before filamentation takes place. Filaments are thus not the cause of merger. In addition, it is important to note that even if merger were caused by filamentation, this would not constitute an explanation for the occurrence of merger. For then it would remain to explain why circular vortices sometimes form filaments (and would merge) and sometimes do not form filaments (and would not merge).

\section{Acknowledgements}

This research was done during a sabbatical stay at the Department of Mechanical and Aerospace Engineering of UCSD. I gratefully acknowledge the hospitality of Professor Paul Linden and the financial support of the University of California Institute for Mexico and the United States (UC MEXUS). I am also thankful for the comments of two anonymous referees, which led to improvements of the paper.

\section{Appendix A. Accuracy of the vortex-in-cell model}

The accuracy of the simulations was monitored by computing the evolution of the following (nominally preserved) quantities: (a) the integral moments of the vorticity distribution $M_{x}=\int x \omega \mathrm{d} a, M_{y}=\int y \omega \mathrm{d} a$ and $M_{x y}=\int\left(x^{2}+y^{2}\right) \omega \mathrm{d} a$, (b) the kinetic energy $K=$ $1 / 2 \int\left(u^{2}+v^{2}\right) \mathrm{d} a$, and (c) the enstrophy $E=\int \omega^{2} \mathrm{~d} a$. As an example, Fig. A.1 shows the time evolution of the error in the conservation of these properties for the unstable Kirchhoff vortex discussed in Section 3. The error at time $t$ is defined as $\Delta X(t)=(X(t)-X(0)) / X(0)$, where $X(t)$ is the value of the property at time $t$ and $X(0)$ is its value in the initial condition. The smallest errors occur in the moments of the vorticity distribution, whereas the largest ones occur in the enstrophy, which decreases by about $1.5 \%$ in the period discussed here.

The numerical dissipation of the simulations at time $t$ may be quantified by computing an effective Reynolds number (see Koumoutsakos, 1997):

$$
\operatorname{Re}^{\mathrm{eff}}(t)=\frac{4 t \Gamma^{2}(t)}{M_{x y}(t)-M_{x y}(0)}
$$

where $\Gamma$ is the total circulation of the flow. This gives a $R e^{\text {eff }}$ of the order of 100,000 in the times discussed here. 


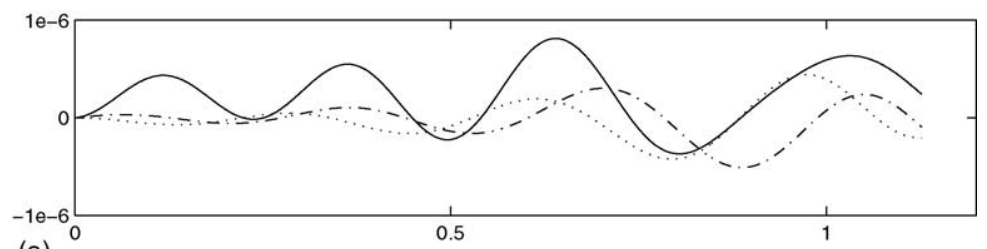

(a)

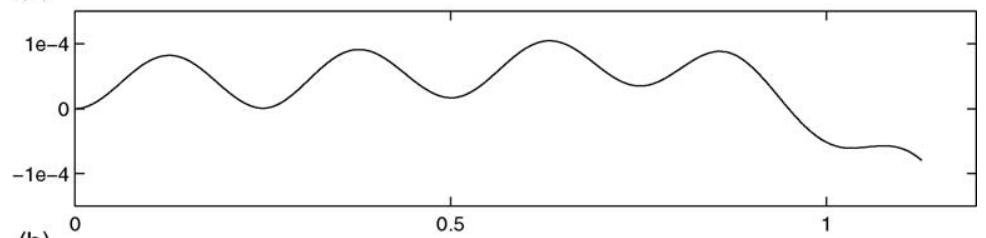

(b)

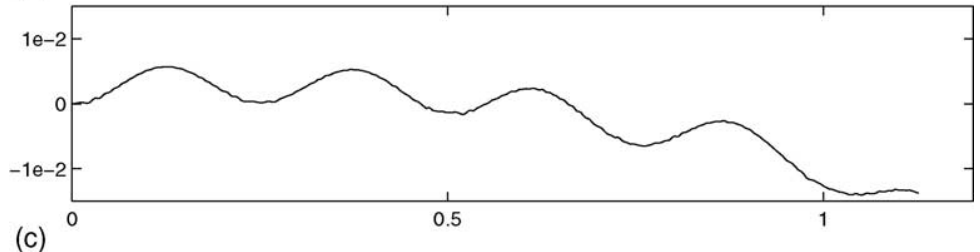

Fig. A.1. Evolution of (nominally preserved) quantities during the evolution of a perturbed Kirchhoff vortex modelled by the vortex-in-cell method (Fig. 3). (a) Moments of vorticity: $M_{x}$ (dash-dotted line), $M_{y}$ (dotted line), and $M_{x y}$ (continuous line); (b) total kinetic energy $K$; and (c) total enstrophy $E$. The horizontal axis gives the time in units of the rotation period of the unperturbed Kirchhoff vortex.

Therefore, it may be concluded that the vortex-in-cell model accurately computes the inviscid dynamics of 2D vortical structures, at least for the periods considered in the present paper.

\section{References}

Beigie, D., Leonard, A., Wiggins, S., 1994. Invariant manifold templates for chaotic advection. Chaos, Solitons Fractals 4, 749-868.

Cerretelli, C., Williamson, C.H.K., 2003. The physical mechanism for vortex merging. J. Fluid Mech. 475, 41-77.

Haller, G., Poje, A.C., 1998. Finite time transport in aperiodic flows. Physica D 352-380.

Hockney, R.W., Eastwood, J.W., 1981. Computer Simulation Using Particles. McGraw-Hill.

Koumoutsakos, P., 1997. Inviscid axisymmetrization of an elliptical vortex. J. Comput. Phys. 138, 821-857.

Malhotra, N., Wiggins, S., 1998. Geometric structures, lobe dynamics, and lagrangian transport in flows with aperiodic time-dependence with applications to Rossby wave flow. J. Nonlin. Sci. 8, 401-456.

Mariotti, A., Legras, B., Dritschel, D.G., 1994. Vortex stripping and the erosion of coherent structures in twodimensional flows. Phys. Fluids 6, 3954-3962.

Melander, M.V., McWilliams, J.C., Zabusky, N.J., 1987. Axisymmetrization and vorticity-gradient intensification of an isolated two-dimensional vortex through filamentation. J. Fluid Mech. 178, 137-159.

Melander, M.V., Zabusky, N.J., McWilliams, J.C., 1988. Symmetric vortex merger in two dimensions: causes and conditions. J. Fluid Mech. 195, 303-340.

Mitchell, T.B., Driscoll, C.F., 1996. Electron vortex orbits and merger. Phys. Fluids 8, 1828-1841. 
Poje, A.C., Haller, G., 1999. Geometry of cross-stream mixing in a double-gyre ocean model. J. Phys. Oceanogr. 29, 1649-1665.

Polvani, L.M., Flierl, G.R., Zabusky, N.J., 1989. Filamentation of unstable vortex structures via separatrix crossing: a quantitative estimate of onset time. Phys. Fluids A 1, 181-184.

Rom-Kedar, V., Leonard, A., Wiggins, S., 1990. An analytical study of transport, mixing and chaos in an unsteady vortical flow. J. Fluid Mech. 214, 347-394.

Velasco Fuentes, O.U., 2001. Chaotic advection by two interacting finite-area vortices. Phys. Fluids 13, 901-912.

Waugh, D.W., 1992. The efficiency of symmetric vortex merger. Phys. Fluids A 4, 1745-1758. 